\title{
Dispersive Raman microscopy
}

\author{
H. Boyer and J. Oswalt
}

Jobin Yvon, Groupe Horiba, 16-18, rue du Canal, 91165 Longjumeau Cedex, France

\begin{abstract}
A survey of dispersive Raman microscopy is presented, together with the newest instrumental configurations, describing the versatility of possible choices of Raman microprobes. Selected applications from various fields of interest are chosen to illustrate the technique, to emphasize concepts like confocality, imaging, selection of excitation laser lines and to discuss their advantages.
\end{abstract}

\section{Introduction}

The multichannel dispersive Raman microprobes have been existing now for nearly a quarter of century [1,2]. Exciting molecules with a laser beam through a confocal optical microscope coupled to a conventional spectrometer, offers the possibility to analyse micro quantities of materials, without any sample preparation and contact and also without any destruction. The lateral resolution, which can be achieved is in the order of magnitude of a micron $(1 \mu \mathrm{m})$, depending on the laser wavelength and the numerical aperture of the microscope objective. The axial resolution at high magnification is better than 2 microns $(2 \mu \mathrm{m})$.

This microscopy technique can be applied on very different types of compounds as gases, liquids and solids, but it is essential to remember that Raman spectroscopy permits to analyse only molecules and structures and not isolated ions. For example, the selection rules demonstrate that centrosymmetric crystals are not Raman "active".

\section{Instrumentation}

Two different types of Raman microprobes are existing, based on the use of either a dispersive spectrometer or a Fourier transform interferometric system.

A dispersive spectrometer is equipped with diffraction gratings, which are covering a very broad range from UV to NIR, when a FT Raman instrument which heart is a Michelson interferometer, is usually limited to only one laser excitation wavelength at $1064 \mathrm{~nm}$. Such a high wavelength is often exciting less fluorescence, but unfortunately is limiting very severely the lateral and axial resolutions of the microscope. It is why the application fields of both techniques - dispersive and FT Raman - do not overlap so much, and most applications are realized with UV, visible or NIR laser wavelengths and then with grating instruments.

These dispersive microprobes are available under a large range of different configurations, from highly versatile triple spectrographs to systems fully dedicated to specific applications like Carbon or On-line Eluxyl analysers.

\section{The laser}

One of the major advantage of dispersive Raman microscopy is that it offers the possibility to select the best laser excitation, depending on all the parameters which permit to record the best Raman information. Among these parameters we will notice the laser power, as Raman signals are proportional to it, the wavelength of this laser excitation, for rejecting fluorescence and thermoemisssion backgrounds or for being under resonance conditions, and the laser emission either continuous or pulsed, this latter one being required for time resolved measurements.

Nowadays, it is possible to use laser lines from UV, down $200 \mathrm{~nm}$-, up to the infrared $1.06 \mu \mathrm{m}$ Nd-Yag laser line, on a power range from microwatts up to several watts.

Considering that the laser beam is focused, in a Raman microscope, on a spot diameter of 1 to 2 microns, the density of the energy received by the sample is for the same laser power much higher than when using a classical macro configuration, and often above the limit temperature for keeping the sample stable. The conclusion is, in these conditions, to go to lower powers, which are available with lower cost devices usually not requiring water cooling and large electricity consumption. It is why most current analytical Raman microprobes are delivered with either $\mathrm{He}-\mathrm{Ne}$, or air - cooled argon or solid state lasers, providing less than $50 \mathrm{~mW}$ exit power and keeping the systems in a reasonnable price range.

\section{The detector}

Most of the current dispersive Raman microprobes are now equipped with multichannel two-dimensional CCD detectors. The main advantages of these detectors are, that they have a high quantum efficiency, an extremely low level of thermal noise and a large spectral range. Many different CCD chips exist, but the most common are matrices of $1024 \times 256$ pixels of $26 \times 26$ microns. They are either available in front-illuminated or thinned back-illuminated versions, the thinning of the chip almost doubling the quantum efficiency of the chip in the visible range. Other chips with smaller pixel sizes, for increasing the spectral resolution, 
have also been developed. For example, chips with a matrix of $2000 \times 800$ pixels, $15 \times 15$ microns each are classical.

The improvement of the quantum efficiency of CCD chips at low or high wavelengths is also possible with either open poly or deep depletion chips. The thermal noise of CCDs is very low, and two cooling versions are standard. The most common one is based on a Peltier thermoelectrically air cooled device, keeping the chip temperature at approximately $-70^{\circ} \mathrm{C}$. It is the device recommended for analytical or industrial Raman systems. But for reaching the highest detectivity and for measuring levels of Raman signals down to $0.01 \mathrm{cps}$ (counts per second), it is necessary to cool the chip down $140 \mathrm{~K}$ with liquid nitrogen.

Also, as a CCD detector is a matrix, it offers the unique advantage of collecting signals along two dimensions, which is an interesting feature for mapping applications. For example, and some applications will be presented in a following chapter, the spectral information is spread along the horizontal axis of the chip and the length of a segment on a sample along the vertical axis; every pixel being then the representation of the Raman signal on a specific sample point at a defined wavenumber.

\section{The microscope}

The use of this attachment in a Raman spectrometer has rapidly enlarged the field of applications, in particular on solid state samples. The possibility to better control the homogeneity of compounds at the micron scale has opened to the Raman technique the domain of pollutions analysis in the semiconductors or polymers fields. Its non-destructive capability brought applications in the geology and artworks domains. And the fact that micro quantities have provided as good results - or better - than large quantities few years earlier, has also been very positive for developing the applications on materials of very small sizes (nanotubes, micropollutions, etc.).

Many general features of the microscope, and in particular confocality, laser line scanning and in situ measurements are presented in a following chapter.

A necessary information all users of this Raman attachment have to keep in mind, is that in the case of a microscope, the same objective is utilized for exciting the sample with the laser beam and for collecting the Raman signal. The separation of both, excitation and collection signals is realized by a beamsplitter. This beamsplitter, in a research instrument which will record spectra down a few wavenumbers, or when using high laser powers, has to have a metallic broadband coating. But, in many analytical instruments which will be employed with low laser powers and will not record low frequency bands, this beamsplitter is a notch filter, used in an injection-rejection configuration as shown on figure 1 .

\section{The spectrometer}

The most important performance of a Raman spectrometer is its rejection of the laser excitation signal. When the only

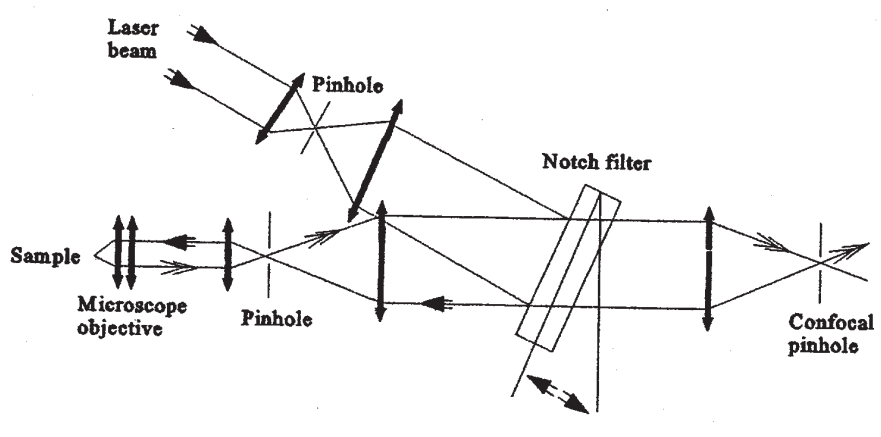

Figure 1. Notch filter used in a injection-rejection configuration.

detector available was a photomultiplier tube, a good Raman spectrometer was requiring several monochromators coupled together, in an additive configuration, for increasing the dispersion and then, by using narrow slits, for better separating the laser line from the low frequency Raman bands.

But, with the introduction of multichannel detectors, which were simultaneously taking signals from a very broad spectral range, it has no longer been possible to use narrow slits and the previous "additive" configuration has no longer been adequate for eliminating the Rayleigh scattering line. The solution for eliminating this very high signal has been to mount in front of a dispersing stage a good filter, consisting in a double subtractive monochromator. Its configuration permits to select on an intermediate slit a spectral bandpass, which wavelengths are all mixed without dispersion on its exit slit. This is the best existing bandpass filter. It is why the best and versatile research Raman spectrographs are conceived around a triple monochromator configuration, the double foremonochromator in a subtractive configuration for rejecting the laser line and the third stage being the dispersing stage of the instrument.

The highest performance triple spectrographs offer additionally to the triple subtractive configuration the triple additive configuration, and also the single spectrograph configuration like analytical systems, for increasing throughput when measuring signals away from the low frequency range.

In the past, single spectrographs could not be used for Raman applications as they were not offering any filtering function. But, since about 15 years ago, new filters called "notch" have been introduced which are totally reflecting a very narrow range, centered on a specific wavelength. When this "cut-off" band is centered on the laser line, a notch filter can easily reflect most of the photons of this line and transmit only the Raman photons. However, as the cut-off shape of a filter cannot be rectangular like the one of a double subtractive monochromator, a notch cannot offer the possibility to record a Raman signal below approximately $100 \mathrm{~cm}^{-1}$. 
The use of notch filters, together with CCD detectors, has been the necessary step for introducing integrated bench-top Raman microprobes which are nowadays commonly present in analytical or control laboratories, and are considered as important as other techniques like FTIR for example.

\section{Specific features and applications}

\section{The confocality}

The developments of the confocal optics in the Raman microprobe have improved the capabilities for the microanalysis of polymers and other transparent or translucient samples.

A confocal device includes two filtering pinholes, the first one located on the laser beam for improving its Gaussian shape, and the second in an exit focal plane of the microscope, for improving both lateral and axial spatial resolutions. Light from out of focus planes being blocked by the adjustable exit pinhole, the lateral and the depth resolutions are strongly enhanced. The magnification and the numerical aperture of the objective used for the analysis and the optical properties of the sample affect the final result. Benefits of the use of confocal optics are also the better discrimination of Raman signals originating from multiple layers, the improved detection of subsurface species showing very weak Raman spectra and the rejection of non specific sample fluorescence. Distribution gradients of molecular species, together with their distribution maps, can be easily set up now and these new arrangements enable research projects, which were not feasible before. Single monolayers deposited on a solid substrate or spread at the air-water interface are studied with confocal optics [3] and Raman spectra of soap films which are constituted of an interstitial water layer and two surfactant monolayers have been presented for the first time in 1998 [4].

In electrochemistry, where the localization and the characterization of chemical species are of concern, the structural changes of a lithium salt with its diffusion depth through a polymer could be clearly demonstrated in the case of rechargeable lithium batteries [5]. It was shown that confocal Raman microscopy is well adapted to in situ investigations on a working polymer electrolyte based battery [68]. Salt concentration gradients inside the electrolyte can be evaluated, polluting species are detected and spectral changes occurring during cycling can be followed. In ref. [9], the technique is applied to the in situ probing of interfacial processes in pulsed-current copper electrodeposition, where it provides the time resolved characterization of the vibrational spectra of the present species by their real time information contents. For other application fields like biology and medicine, confocal Raman microscopy enables new insights as it was demonstrated for example by the large amount of work performed by a research group in the Netherlands [10].

Representative spectra of a depth profiling through a polymer sample are shown in figure 2 . The sample is a $75 \mu \mathrm{m}$ thick layered polymer film. The four spectra have been recorded at the upper surface of the film where a Raman spectrum of pure polyethylene (top left) was obtained, from a layer about $20 \mu \mathrm{m}$ inside the film showing a mixed spectrum of polyethylene and nylon (top right), from the middle layer with a spectrum of pure nylon (bottom left), and from the layer at the lower surface, recorded through the entire film depth with a nearly pure spectrum of polyethylene (bottom right). The differences between all of them are best seen looking at the line above $1600 \mathrm{~cm}^{-1}$. These results were obtained in less than two minutes. Without the confocal capabilities of the Raman microprobe, only the spectrum of the mixture of these compounds would have been obtained.

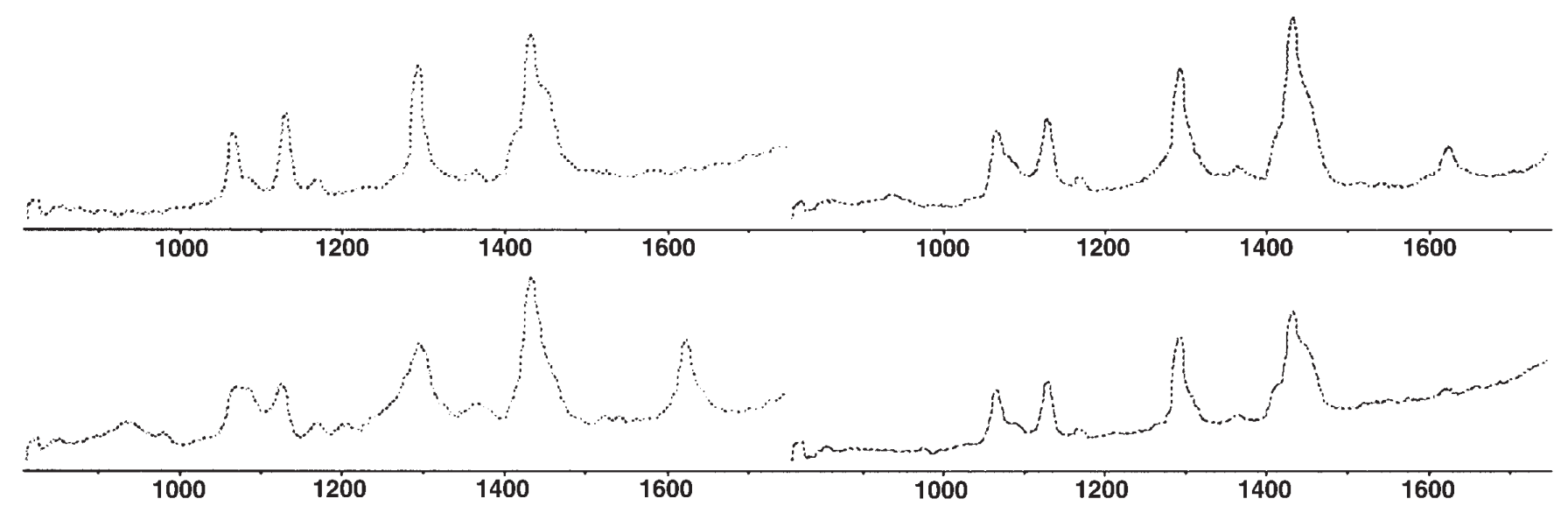

Figure 2. Raman spectra of a depth profiling through a $75 \mu \mathrm{m}$ thick polymer film. 


\section{Optical fibers}

Optical fibers as probes for Raman experiments have been used for more than fifteen years. What is less known is that Raman microscopy can also be performed at distance using optical fibers and portable accessories, specially developed for these applications. Usually these accessories include a camera and a microscope objective in order to visualize the very small objects to be analyzed, which are in general part of much bigger ones that cannot be placed under a standard microscope. Expertises such as pigment identifications by Raman microscopy have recently gained much interest for museology and archaeology. Correct identification is critically important in restorative work, since restauration with incorrect pigments could lead to chemical reactions between incompatible pigments or simply to some incorrect hue. Conservation studies can require the identification of pigment degradation products and the development of procedures for pigments conservation.

\section{Raman imaging}

A motorized $x y z$ microscope stage enables to record automatically spectral files which will constitute the basis of Raman images, Raman maps or collections of Raman spectra recorded on preselected points or averaged zones. Specific software routines will allow the quick and easy reconstruction of these maps, according to some parameters to be selected. The possibility of generating two-dimensional and three-dimensional images of a sample, using various spectral features, is an evident advantage over either traditional spectroscopy or microscopy.

Pioneer work was accomplished by a French research team in Reims [11 and ref. therein). Working in the biology and medicine field, they feel for example, that one of the most promising applications of this method is the study of the distribution and of the mechanisms of the antitumor drugs action inside single living cells. A commercial sheet of a refractory ceramic composite material is characterized by Raman microscopy [12] and Raman mapping is employed to spatially resolve the components of the ceramic composite, demonstrating that Raman imaging capabilities bring new insights in totally different domains. Not only the construction of a map has to be considered, but also the time saving of the automated procedure or the possible discovery of unexpected results during the rapid inspection of the more or less large set of individual spectra. The following example from the pharmaceutical domain illustrates the interest of a distribution map and of the averaged spectrum over a whole image. The Raman spectra of a pure tablet components i.e. lactose and sulfonilamide are shown in figure 3. As these spectra are very different, these two compounds are easily distinguished. For the reconstruction of the distribution map shown in figure 4 , an optimal step size of $2 \mu \mathrm{m}$ was determined from the averaged grain size of non compressed powders. The surface which was scanned was $100 \mu \mathrm{m} \times 100 \mu \mathrm{m}$. On this picture, bright areas correspond to lactose, the excipient, whereas the dark areas especially in the middle and on the left side show sulfonilamide

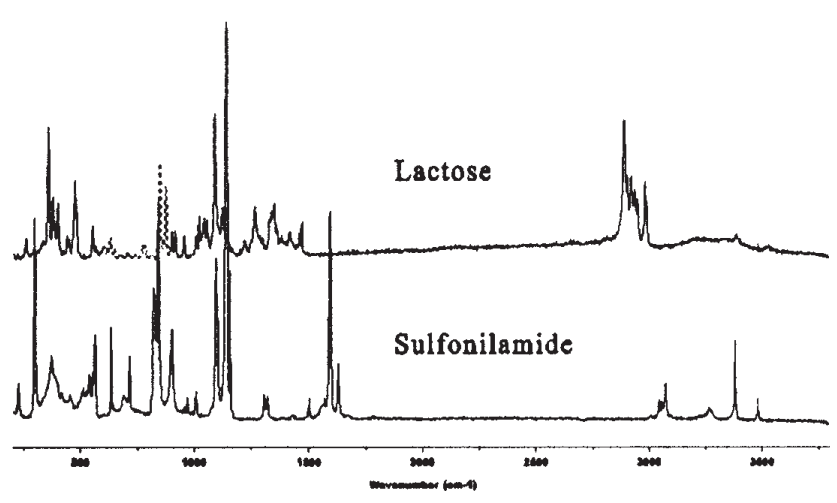

Figure 3. Raman spectra of the tablet components.

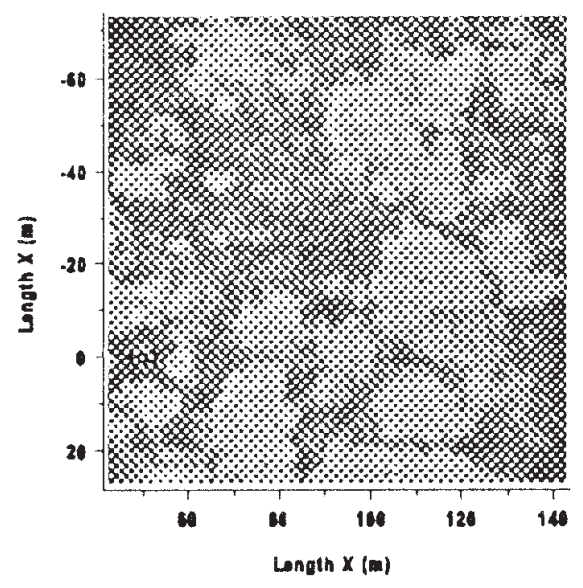

Figure 4. Raman distribution map of the tablet components.

aggregates. On this black and white picture, some very small mixed areas are not visible. The purity on each point of the sample can be checked looking at individual spectra, but they are not shown here. Figure 5 shows a map of the distribution of the same compounds, but now over half a tablet i.e. over a surface of approximately $4 \mathrm{~mm} \times 10 \mathrm{~mm}$. On such distances, as the tablet was curved, a lower magnification objective was used together with a longer step size of $100 \mu \mathrm{m}$. It can be seen from this picture that no compound is agglomerated at a particular location. The spectrum on figure 6 represents the averaged spectrum calculated over the surface mapped in figure 5. Calculations for quantitative purposes can be derived from this type of averaged spectra, which can cover any defined size on a Raman image.

Since the beginning, an application field of choice has been found for the Raman microprobe in geology and mineralogy and up to now GeoRaman conferences have been hold periodically. The non-destructive identification of fluid 


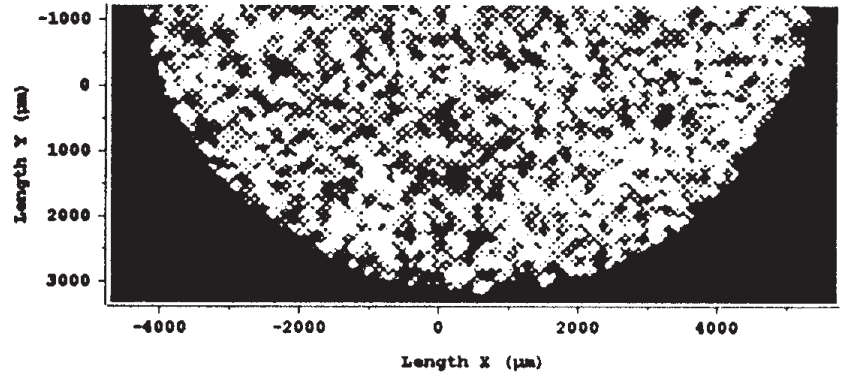

Figure 5. Raman distribution map over half a tablet.

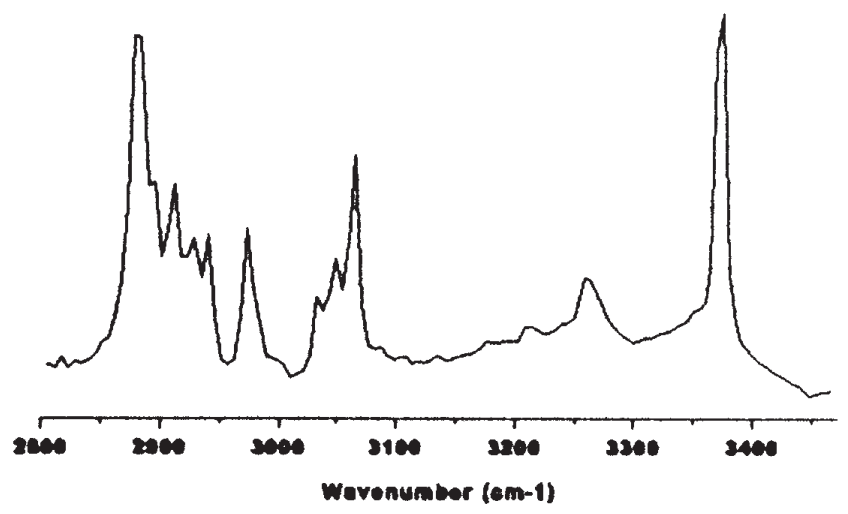

Figure 6. Mean Raman spectrum over figure 5.

ple point. In this case for example, the ease of obtaining an averaged information reveals all its interest. An accessory called line scanning offers another possibility to obtain a mean spectrum along a line, (as well as the spectra of individual points along this line) without a motorized microscope stage. The line scanning allows also getting a quick overview of the major constituents of a sample in a real time display.

\section{Various excitation wavelengths}

A classical Raman spectrum is known to do not change whatever the wavelength of the exciting laser line, and this property even constitutes a test for distinguishing a Raman line from a narrow fluorescence band. Large fluorescence bands responsible for high backgrounds or to the worst, of the obturation of the Raman signal, are more or less cir-

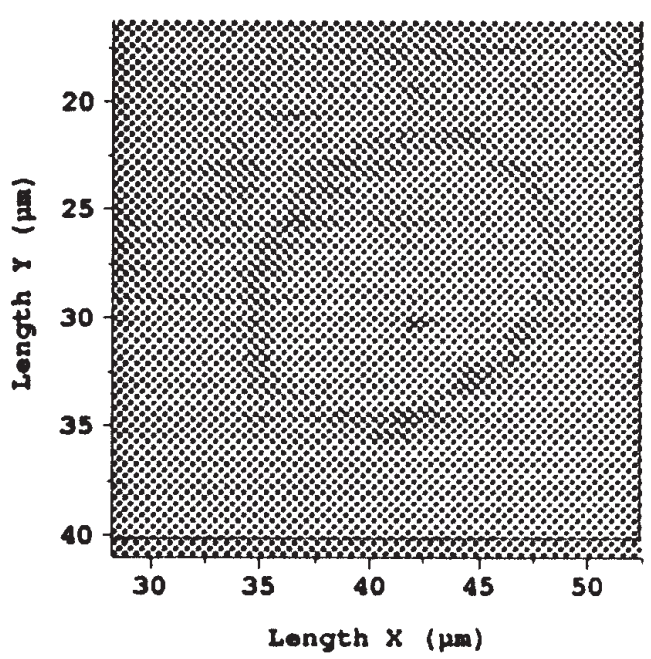

Figure 7. TV image of a solid inclusion.

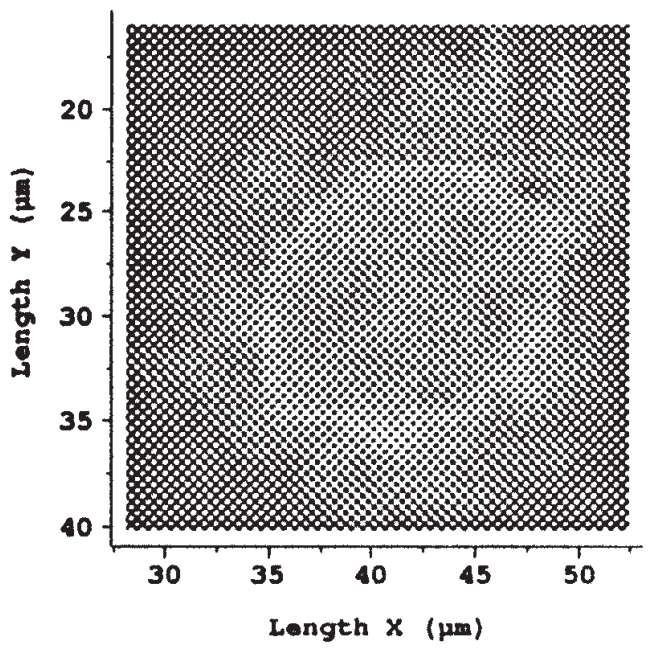

Figure 8. Raman image of the inclusion in figure 7. 
cumvented by the change to a more adequate exciting line. The easy change of the exciting laser line is suitable when doing Resonance Raman Spectroscopy, where the position of the absoption band of a molecule or a chromophore relatively to the exciting laser line may produce an enhancement of the Raman spectrum by several orders of magnitude. The penetration depths in semiconducting materials can be adjusted by the change of the laser wavelength. Even from now analytical instruments with two built-in lasers allow working from UV up to near IR (244 nm to $785 \mathrm{~nm}$ ) with a microscope, only by switching from one laser to the other one. The Raman spectrum on figure 9 is obtained from an ordinary teflon screw (polytetrafluoroethylene) in $20 \mathrm{sec}-$ onds with a $244 \mathrm{~nm}$ excitation and a UV optimized microscope objective.

$\mathrm{GaN}$ is a sample from the semiconductor industry, and its micro photoluminescence and Raman spectrum with a UV excitation is shown on figure 10. Figure 11 is an expanded view of figure 10, where the Raman part with its overtones is more visible. If the photoluminescence signal falls inside the spectral range of the CCD, then an analytical instrument as used here is adequate. But if another detector in the infrared range is needed, then a versatile system becomes necessary, with a supplementary exit for a second detector. In any case, the two spectra can be recorded from the same sample point, in order to benefit of the complementary information content of these two techniques.

The carbon industry has become a major applications field for Raman microscopy, which is widely employed for the characterization of different types of carbon materials. The possibility to use easily various excitation wavelengths

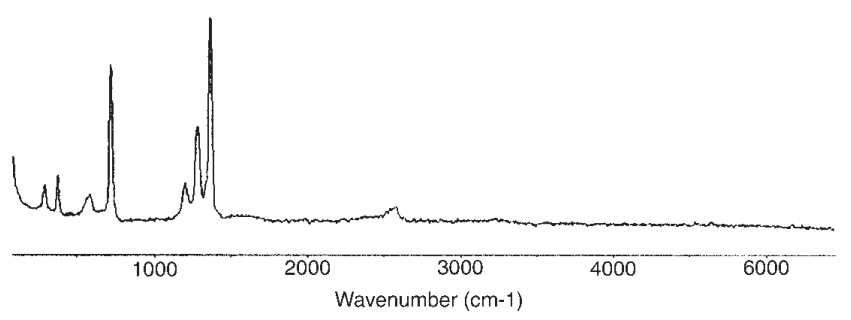

Figure 9. UV exited micro Raman spectrum. is an important factor, as the changes observed in the Raman spectra are used to quantify the different carbon types present in the sample. For example, spectra of carbon materials with excitations at 514.5 and $632.8 \mathrm{~nm}$ of carbon materials are shown in ref. 13, which permit to determine nanocrystalline graphite.

Many useful data of Raman spectroscopy and especially the penetration depths in semiconductor materials versus the laser wavelength will be found in ref. [14].

\section{Low frequency measurements}

As mentionned before, measurements in the low frequency range require to use a triple system in a subtractive configuration, which guarantees the recording of Raman spectra closest to the laser line. A typical application is the measurement of LAM modes (Longitudinal Acoustic Modes), occurring in superlattices produced by the semiconductor industry. LAM modes may be observed as well in some polymer or oligomer samples. These modes are in the direct vicinity of the laser line, between 4 to $50 \mathrm{~cm}^{-1}$ so that the highest stray light rejection, only provided by an excellent foremonochromator, is required to eliminate the laser light. It is also to notice that with a microscope, the laser light reflected by the sample enters directly the monochromator, increasing thus the difficulty for its discrimination, as compared to a macro sampling device where the reflected beam can be directed outside the solid angle of the collecting optics. If the study of lattice modes which occurs between 20 to $200 \mathrm{~cm}^{-1}$ or above are of concern, the use of the same type of instrument is recommended, because of the totally flat response of the system in this region.

The LAM modes observed from superlattices give information on their quality. Figure 12 shows a Raman spectrum of a germanium - silicon superlattice, where the pure $\mathrm{Ge}$ mode is located at $\sim 300 \mathrm{~cm}^{-1}$, the pure Si mode at $521 \mathrm{~cm}^{-1}$, the perturbed $\mathrm{Si}$ mode at $\sim 510 \mathrm{~cm}^{-1}$, and the mixed $\mathrm{Ge}-\mathrm{Si}$ mode in between them, all being phonon modes. Figure 13 is the expanded part very close the laser line of the LAM modes shown on figure 12, their features attesting the excellent quality of this superlattice, where the lowest line is situated at $4.7 \mathrm{~cm}^{-1}$. The lattice constant can be derived from the interval between these lines.

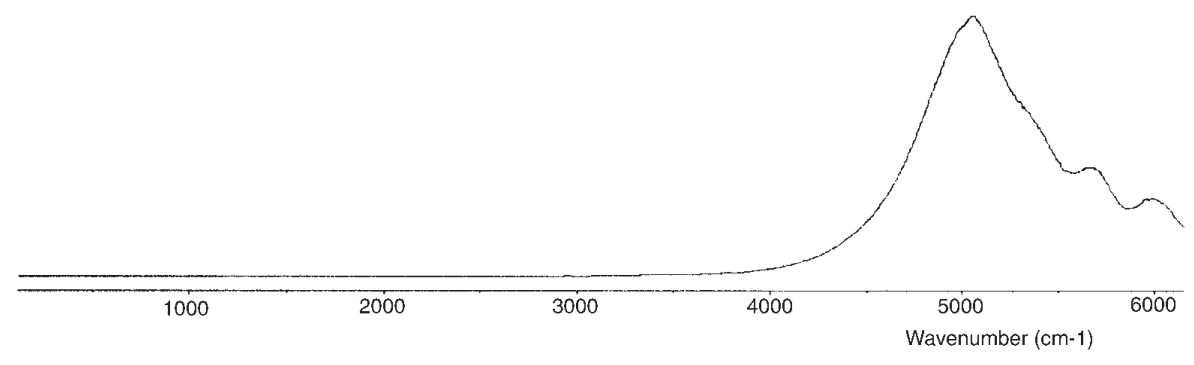

Figure 10. Photoluminescence and Raman spectrum of GaN excited at $325 \mathrm{~nm}$. 


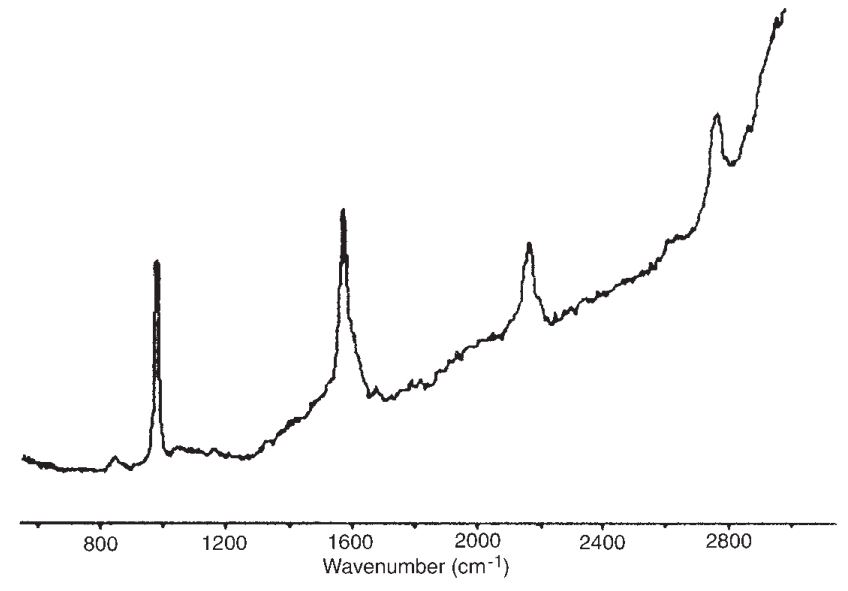

Figure 11. Expansion of figure 10.

\section{Stress measurements}

A stress in polymers or carbon fibers, freestanding or imbedded in polymer matrices, is usually revealed by a frequency shift over several wavenumbers, which means that an analytical instrument is performant enough for these measurements. Sometimes, for the determination of a stress in semiconductors, the highest dispersion is required as in these materials the line shift can affect only tenths of wavenumber. For this case, a choice exists between the additive configuration of a triple system and a system dedicated to the measurement of line shifts. The first one would be preferred probably when stress measurements occur occasionnally in the laboratory, whereas the dedicated system is better suited for an every day repetitive task. In reference [15], strain in GaN layers as well as local changes in orientation and free carrier variations have been evidenced when approaching the substrate interface. For some measurements, cooling the sample became necessary, and was performed in a microscope cryostat. In reference [16], the simplicity of the experimental procedure, i.e. the combination of micro-Raman spectroscopy with indentation tests, was pointed out as a fast tool for monitoring pressure induced stresses and phase transitions in materials. The formation of silicon polymorphs other than amorphous or metallic structures during hardness intentation was reported here for the first time. Microscopic strain distributions in varying the thickness of polysilicon layers deposited as bridges over silicon substrates were determined by high resolution micro-Raman measurements. Shifts in Raman band frequencies as a function of their position on the brigde structures were observed to be dependent on the thickness of the membrane layer as well as the annealing conditions [17].

Because of an understandable lack of place in this application part, only some examples have been explained and mentionned and the cited references were only chosen in order to provide an enlargement of ideas on a minimum space. Many other works of high interest and high quality were performed recently in the field of Raman microscopy and we apologize for not mentionning all of them.

\section{Conclusion}

Raman microscopy is not yet a totally push - button technique, and that is the reason why some indications on the
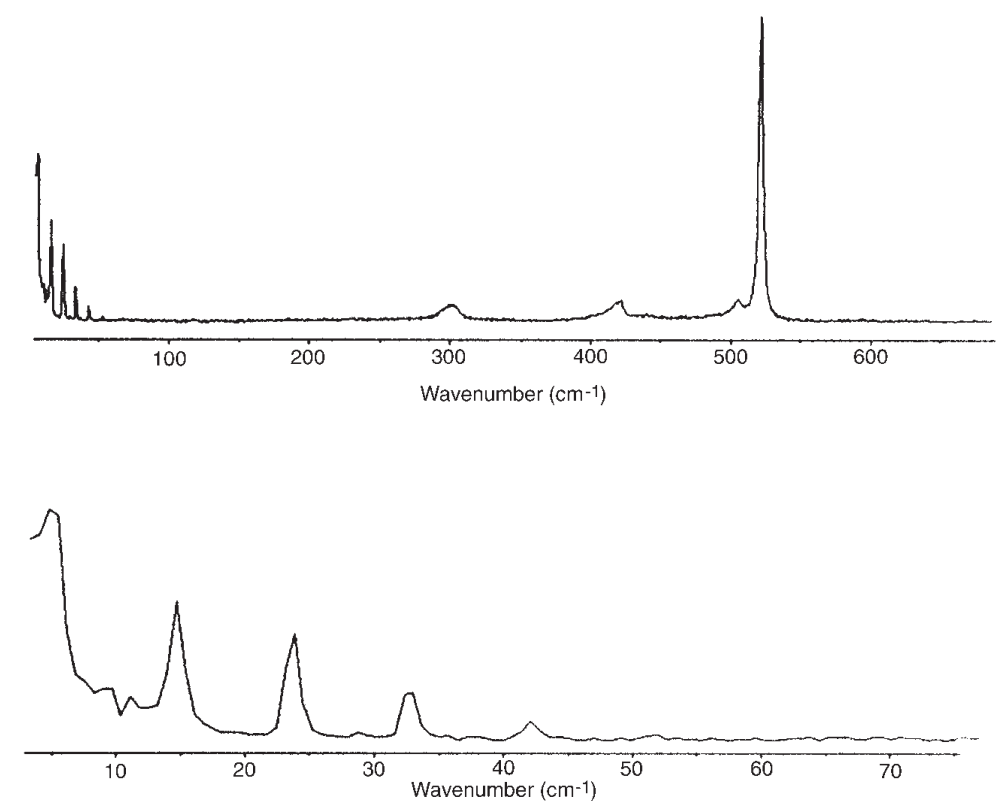

Figure 12. Raman spectrum of a $\mathrm{Ge}-\mathrm{Si}$ superlattice.

Figure 13. LAM modes of figure 12. 
instrumentation are proposed in this paper. In the applications part, only a few recent applications have been mentionned, but we hope that they have demonstrated that Raman microscopy is already a very useful technique for many applications fields and has enough unique advantages for becoming soon an inescapable tool in all research, analytical and control laboratories.

\section{References}

1. Delhaye, M.; Dhamelincourt, P. J. Raman Spectrosc. 1975, 3, 33.

2. Rosasco, G.J.; Etz, E.S.; Cassatt, W.A. Appl. Spectrosc. 1975, 29, 396.

3. Castings, N.; Blanchez, D.; Desbat, B.; Turlet, J.M. Thin Solid Films 1996, 284, 631.

4. Lecourt, B.; Capelle, F.; Adamietz, F.; Blanchez, D.; Kellay, H.; Turlet, J.M. J. Chem. Phys. 1998, 108,1284.

5. Fran Adar, Pers Comm.

6. Rey, I.; Lassègues, J.C.; Baudry, P.; Majastre, H. Electrochim. Acta 1998, 43, 1539.

7. Rey, I.; Bruneel, J.L.; Grondin, J.; Servant, L.; Lassègue, J.C. J. Electrochim. Soc. 1998, 145, 3034.
8. Lassègues, J.C.; Bruneel, J.L.; Grondin, J.; Rey, I.; Servant, L.; Vignan, L. Mol. Cryst. Liq. Cryst. 1998, 310, 211.

9. Texier, F.; Servant, L.; Bruneel, J.L.; Argoul, F. J. Electroanal. Chem. 1998, 446, 189.

10. Greve, J.; Otto, K.; Puppels, G.J. at Department of Applied Physics, Twente University of Technology, Enschede, The Netherlands.

11. Sharnov, S.; Chourpa, I.; Valisa, P.; Fleury, F.; Feofanov, A.; Manfait, M. Microscopy and Analysis 1994, 9; 510, Manfait, $\mathrm{M}$ at Laboratoire de Spectroscopy Biomoléculaire, UFR de Pharmacie, 96 Reims France.

12. Panitz, J.C.; Wokaun, A. Appl. Spectrosc. 1998, 52, 1252.

13. Gogotsi, Y.G.; Jeon, I.D.; McNallan, J. J. Mater. Chem. 1997, 7, 1841.

14. De Wolf, I.; Jimenez, J.; Landesman, J.P.; Frigeric, C.; Braun, P.; Da Silva, E.; Calvet, E. Raman and Luminescence Spectroscopy for Microelectonics, European Communities, 1998, Directorate General XII, EUR 18597.

15. Sigle, H.; Thurian, P.; Eckey, L.; Kaczmarzyk, G.; Filippidis, L.; Hoffmann, A.; Broser, I.; Litvinchuk, A.P.; Thomsen, C. Internat. Symp. on Blue Laser and Light Emitting Diodes, 1996, Chiba, Japan.

16. Kailer, A.; Gogotsi, Y.G.; Nickel, K.G. J. Appl. Phys. 1997, $81,3057$.

17. Talaat, H.; Negm, S.; Schaffer, H.E.; Adar, F.; Nassiopoulos, A.G. Appl, Surf, Sci. 1998, 123/124, 742. 